\title{
Sistema de evaluación sustentado en el conocimiento ancestral para potenciar los aprendizajes en la escuela Evaluation system based on ancestral knowledge to promote learning at school
}

\author{
Mary Lili Caicedo Arroyo \\ mcaicedoarroyo@gmail.com \\ Calidad Educativa \\ Alcaldía de Buenaventura \\ Buenaventura, Colombia
}

\begin{abstract}
Resumen- Las comunidades negras que han habitado el Pacífico medio colombiano se caracterizan por integrar toda la vida, su cotidianidad, cosmovisión, valores tradicionales, formas de interacción y filosofías en torno al relacionamiento con su entorno natural, cultural y social. Así, han integrado de manera natural habilidades que hoy identifican a la escuela del siglo XXI, como son: la colaboración, los buenos liderazgos, la construcción de paz, la empatía, la comunicación efectiva y el respeto por la vida en todas sus formas. Si bien la escuela es una institución liderada por el Estado, ésta debe incorporarse a las dinámicas comunitarias, definir las metas en las que convergen y convertirse en un apoyo para formar seres humanos críticos, creativos y propositivos con una gran capacidad de resiliencia, autodeterminación y pensamiento colectivo para encarar los desafíos y dificultades generadas a raíz del conflicto armado que ha arreciado por décadas en estos territorios. La investigación desarrollada en las comunidades negras del río Yurumanguí, Distrito de Buenaventura, Colombia, ha demostrado que la potencia de la escuela se encuentra sin duda, en la articulación de todos los procesos y la vida comunitaria a la escuela, y esto, a través de los Sabedores, líderes naturales que ejercen un papel formativo desde el ser, y que abre paso a que el conocimiento disciplinar sea comprendido por los estudiantes y estos, a su vez, desarrollen habilidades humanas que les permita forjar la vida en ambientes de construcción social y pacífica, defendiendo el territorio y su acervo cultural, la solidaridad, la autonomía y la capacidad de adaptación en un mundo cambiante.
\end{abstract}

Palabras clave: conocimiento ancestral, etnoeducación, sabedores, principios $y$ valores ancestrales.

Abstract- The ethnic groups that have inhabited the middle Pacific region of Colombia are characterized by integrating their whole life, their daily life, worldview, traditional values, forms of interaction and philosophies regarding their relationship with their natural, cultural and social environment. Thus, they have naturally integrated skills that today identify the school of the 21 st century, such as: collaboration, good leadership, peace building, empathy, effective communication and respect for life in all its forms. Although the school is an institution led by the State, it must be incorporated into community dynamics, define the goals in which they converge and become a support to form critical, creative and proactive human beings with a great capacity for resilience, self-determination and collective thinking to face the challenges and difficulties generated by the armed conflict that has raged for decades in these territories. The research developed in the black communities of the Yurumanguí River, District of Buenaventura, Colombia, has shown that the power of the school is undoubtedly found in the articulation of all the processes and community life to the school, and this, through the Sabedores, natural leaders who exercise a formative role from the being, and that opens the way for disciplinary knowledge to be understood by the students and these, in turn, develop human skills that allow them to forge life in environments of social and peaceful construction, defending the territory and its cultural heritage, solidarity, autonomy and the ability to adapt in a changing world.

Keywords: ancestral knowledge, ethnoeducation, ancestral principles and values, community-school methodological approach.

\section{INTRODUCCIÓN}

La educación es un derecho fundamental en el ser humano, es la oportunidad para impulsar el desarrollo sostenible y avanzar hacia la construcción de paz. Cada uno de los objetivos de la Agenda 2030 requiere como elemento transversal una educación de calidad, equidad y pertinencia, que facilite a los individuos la adquisición de competencias, conocimientos, principios y valores morales que le permitan vivir en condiciones dignas, construir su proyecto de vida desde su individualidad, pero aportando deliberadamente a las sociedades en que habitan.

Según Marles (1997) la educación en los grupos étnicos corresponde a procesos endógenos de formación y socialización, de acuerdo a las características culturales, sociopolíticas, económicas y lingüísticas propias, de tal manera que mediante este proceso permanente se garantice la interiorización del ascendiente cultural que ubica al individuo en el contexto de su propia identidad. Dicho esto, propiciar que los estudiantes se conviertan en los protagonistas de su propio aprendizaje, motivando y gestionando la autorreflexión de los actores educativos como la comunidad y la escuela, articulando el conocimiento ancestral desde sus principios y valores culturales con el disciplinar y enfocar la figura del Sabedor comunitario y el docente como mediadores entre el estudiante y el aprendizaje, es definitivamente el camino para que los niños, niñas y jóvenes, no sólo de grupos étnicos, sino en general, accedan a una educación de vanguardia que responda a las necesidades de sus comunidades, que genere aprendizajes para la vida, pero desde lo cercano, que conserve su identidad y tenga la capacidad de valorar y enriquecer la cultura propia con los 
aportes de otras, en una dimensión de alteridad cultural a partir de un diálogo respetuoso de saberes y conocimientos que dialoguen y se complementen mutuamente.

La Constitución Política de Colombia en su Artículo 7, reza que el Estado colombiano reconoce y protege la diversidad étnica y cultural de la Nación, ello implica, que se debe impulsar una educación intercultural, que favorezca el respeto, la tolerancia, el diálogo y el enriquecimiento cultural como lo reitera Marles (1997), y que desde los territorios étnicos, se aborde un sistema de valoración etnoeducativo que abarque la cosmovisión de los pueblos culturalmente diferenciados, como mecanismo de resistencia, que reduzca la brecha en la calidad educativa, promueva la pervivencia en condiciones dignas y la sostenibilidad de la riqueza cultural.

De la misma manera, la ley 70 de 1993 "reconoce la propiedad colectiva de la tierra de las comunidades afrocolombianas que históricamente han habitado tierras baldías en las zonas rurales ribereñas de los ríos de la Cuenca del Pacífico, de acuerdo con sus prácticas tradicionales de producción, el derecho a la propiedad colectiva". Así mismo, tiene como propósito establecer mecanismos para la protección de la identidad cultural y de los derechos de las comunidades negras de Colombia como grupo étnico, y el fomento de su desarrollo económico y social, con el fin de garantizar que estas comunidades obtengan condiciones reales de igualdad de oportunidades frente al resto de la sociedad colombiana. (Colombia, s.f.)

Procesos de investigación como la que se presenta traduce la normatividad y el reconocimiento del derecho en acciones concretas en la escuela, donde los habitantes de las comunidades se encargan de la educación del ser a través de encuentros intergeneracionales, jornadas escolares de pautas de crianza familiar, donde la escuela es el puente que facilita la comunicación y el diálogo comprensible entre estas dos generaciones. Ello, impulsado desde la escuela y medido a través de indicadores que demuestran el avance en la adquisición de los valores tradicionales que son monitoreados no sólo desde las aulas, sino en la vida cotidiana y en su interacción con la familia y la comunidad.

En razón a lo expresado anteriormente, es evidente que la escuela en estos territorios se convirtió en un aliado en la defensa de la vida comunitaria, formando hombres y mujeres sensibles al dolor del otro, líderes con un alto sentido de pertenencia e identidad que les permite acceder al conocimiento del mundo a través del ancestral.

\section{CONTEXTO}

La iniciativa innovadora que se plantea en este documento se desarrolla desde hace dos años en la Institución Educativa Esther Etelvina Aramburo ubicada en el Consejo Comunitario de la comunidad negra de la cuenca del río Yurumanguí, distrito de Buenaventura, Valle-Colombia. Cuenta con un total de 1.039 estudiantes en sus doce sedes veredales, que se extienden desde la bocana del río hasta la cabecera. Los alumnos son atendidos en jornadas diurnas y nocturnas, en los niveles de prescolar, básica primarias, básicas secundarias y media vocacional, además de la educación para adultos.
La comunidad se encuentra organizada como Consejo Comunitario en el marco de la ley 70 de 1993 que les otorga derechos especiales como grupo étnico.
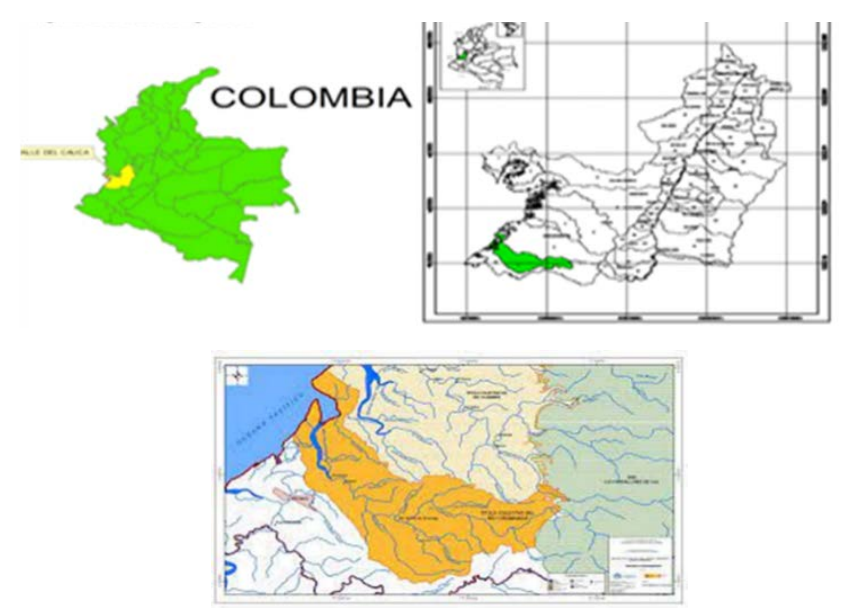

\section{Figura 1.}

Ubicación geográfica de la cuenca del río Yurumanguí. (fuente PMT-Yurumangui)

Según la distribución del Consejo Comunitario, hay trece veredas en las cuales existen 563 familias que contabilizan un total de 2.182 habitantes, que en su conjunto representa al Consejo Comunitario del río Yurumanguí. Datos tomados del auto censo CCY (2020).

Es conveniente mencionar que la comunidad se encuentra avanzando en procesos de reparación colectiva liderada por el Estado a causa de los daños y las secuelas que dejó la incursión de grupos armados al margen de la ley en el tejido social y cultural de sus habitantes. Décadas de conflicto armado dejó rupturas en los diferentes atributos como: prácticas colectivas, formas de organización, formas de relacionamiento, en los liderazgos e identidad.

Algunas consecuencias evidentes hoy en el servicio educativo que reciben los alumnos, tiene que ver con la debilidad en los procesos de educación comunitaria que era responsabilidad de los sabedores o adultos mayores que se configuraban como autoridad moral y organizativa en el colectivo. Esta pérdida de valores trajo consigo múltiples conflictos relacionados con el aspecto ambiental, de relacionamiento, pérdida del valor a la palabra dada, monetarización de la vida, de la solidaridad, de las pautas de crianza y el pensamiento desde el bienestar colectivo antes que el individual.

En razón a lo anterior, los resultados en las pruebas saber 11 evidencian que la institución está un $25 \%$ por debajo de la media nacional, lo que aumenta la brecha de la desigualdad al no poder acceder a más y mejores oportunidades laborales y académicas. 


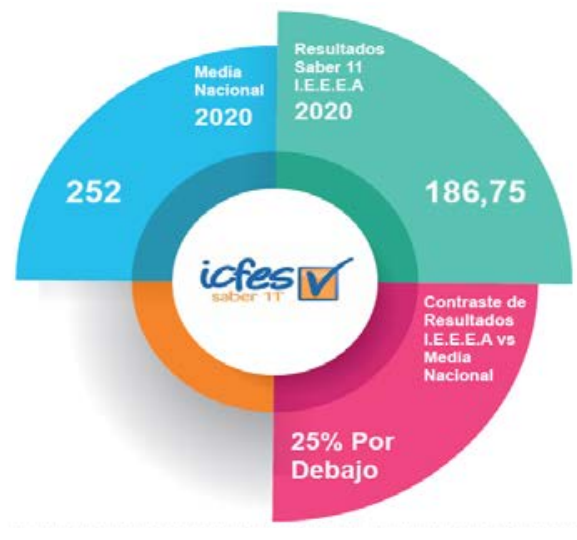

Figura 2.

Contraste de resultados Pruebas de Estado (Saber11)

De acuerdo con el estudio realizado, una de los principales necesidades en la escuela rural del Pacífico medio en Colombia, deriva en la débil contextualización del sistema educativo al medio cultural y social de la comunidad; así, estas poblaciones son débilmente abarcadas por las políticas educativas, bien sea por el difícil acceso debido a su ubicación geográfica o por las dificultades relacionadas con el conflicto armado en la zona; sin embargo, la comunidad-escuela trabajando de manera articulada en las aulas y en la vida cotidiana (fuera de la escuela), sacando las clases de las aulas y llevándolas a la comunidad, ha sido el camino más expedito para lograr la preservación de los mecanismos de resistencia ante la adversidad, la transmisión del conocimiento ancestral y la intervención de la comunidad en la formación de seres humanos integrales.

Por ello, se enfocó la investigación-intervención a partir de la articulación de los conocimientos ancestrales con los aprendizajes de los alumnos de la comunidad focalizada, que permitan un acompañamiento más acertado a los alumnos, con clases que se enfocaran en la exploración del entorno y sus riquezas y aprovechando los recursos y oportunidades de aprendizaje a través de lo propio.

\section{A. Objetivos}

General: incorporar las prácticas culturales ancestrales de la comunidad a la escuela para favorecer el aprendizaje disciplinar y potenciar el ejercicio del ser como generadores de paz y sana convivencia en los alumnos de la escuela Esther Etelvina Aramburo del área rural del Distrito de Buenaventura, Colombia.

\section{Específicos:}

Describir la manera en que las experiencias pedagógicas desde los entornos comunitarios favorecen el ejercicio de los buenos liderazgos, la participación y la identidad en los alumnos.

Demostrar cómo el propiciar espacios de acercamiento entre los sabedores y conocedores ancestrales con las nuevas generaciones facilita la transmisión del conocimiento ancestral y potencia el disciplinar.
Describir la metodología que integra a los sabedores comunitarios en su rol de mediadores y la planificación curricular en conjunto con los docentes de aula.

Describir la aplicación del enfoque etnoeducativo de comunidad-escuela, concepto planteado por Riascos (2021), como mecanismo para contextualizar la educación.

Para la iniciativa es importante la participación activa de los aliados y beneficiarios en razón a que cada grupo tiene unos roles definidos que al interactuar desde sus fortalezas y oportunidades impulsan la trazabilidad en el proceso innovador. Entre ellos podemos mencionar los siguientes:

Estudiantes: al ser impactados directamente por la estrategia, pueden aportar a la gestión de calidad del proyecto en aras de lograr el mejoramiento continuo; el proyecto les aporta nuevas ideas $\mathrm{y}$ elementos de autogestión del conocimiento $\mathrm{y}$ aprendizajes significativos.

Docentes -mediadores: son aliados de mucha influencia, pues establecen los puentes a través de la coordinación de actividades conjuntas con los Sabedores culturales de la comunidad. Aportan muchos conocimientos e ideas de mejoramiento; gracias a su experiencia de aula, son cruciales a la hora de motivar a los alumnos e implementar de manera óptima los recursos y estrategias acordes a las necesidades de los alumnos. El proyecto les aporta nuevos conocimientos y competencias en el marco de la educación activa del siglo XXI.

Comunidad: para efectos del proyecto en general, la comunidad es uno de los actores más importantes, pues son sus sabedores los que validan, apoyan y co-evalúan el accionar de los alumnos en la comunidad, respaldan y acompañan al proceso de implementación del proyecto, pues hablamos de una "comunidad gestora del conocimiento". Aportan al proyecto ideas y difusión y obtienen elementos para la recuperación de tejido social y cultural, y actualización al seguimiento del aprendizaje de los alumnos.

Asesor Pedagógico Externo: es un aliado de alta influencia, aporta la conceptualización y orientación y promueve la cocreación. Se beneficia ganando experiencia al participar del diseño e implementación de una iniciativa innovadora, que rompe paradigmas y que aportará a la construcción de país.

Como beneficiarios indirectos se encuentra toda la comunidad del Consejo Comunitario de la cuenca del río Yurumanguí, de la región y en general de todos los grupos étnicos del país que comparten las mismas necesidades en materia educativa y cultural. En suma, el bienestar colectivo depende de que se generen las condiciones para una educación con calidad, equidad y pertinencia, que garantice el desarrollo armónico del territorio y sus condiciones de vida futuras. 


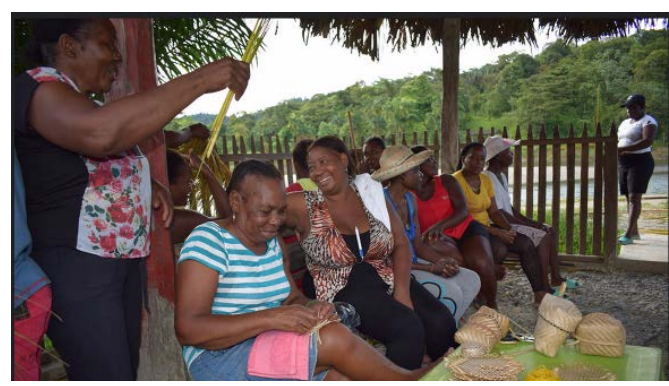

Figura 3.

Grupo de conocedoras y sabedoras artesanas vereda San Antonio

\section{DESCRIPCIÓN}

Se desarrolla una iniciativa de transformación educativa, a través de la inclusión del rol de los sabedores comunitarios dentro del sistema de evaluación institucional que permite valorar, no sólo competencias académicas, sino también la incorporación de la práctica de principios y valores ancestrales co-evaluados y respaldados por los conocedores y sabedores comunitarios.

En otras palabras, se responde a la pregunta de investigación “¿Cómo dinamizar los aprendizajes para la vida a través de la incorporación del saber ancestral al sistema de evaluación educativo de manera que fomente los principios y valores ancestrales en los alumnos para favorecer la paz y la sana convivencia en el entorno escolar y comunitario?"

Esta pregunta de investigación se ha ido construyendo desde hace dos años y dando elementos claves para obtener su respuesta acorde con las realidades existentes al interior del territorio.

Dentro del desarrollo de las actividades realizadas por la comunidad-escuela se llevan a cabo jornadas de trabajo que involucran a los médicos ancestrales como parteras, hierbateros y sobadores, artesanos, autoridades eclesiales del territorio que le dan el sentido espiritual a la vida, los pescadores, carpinteros, entre otros. Este grupo al que llamamos Sabedores representan una autoridad tradicional a la que los niños y jóvenes manifiestan respeto y reconocimiento como autoridades morales. De esta manera, los Sabedores aportan sus conocimientos en las clases que son previamente planificadas entre el docente mediador y los sabedores, procurando establecer el nexo con las temáticas desarrolladas y de esta manera, generar aprendizajes que abordan el reconocimiento del mundo real, iniciando por lo que tienen cercano.

Un ejemplo palpable de estos encuentros es el del abordaje de los ejes temáticos relacionados con la sostenibilidad ambiental; en éste ámbito el docente mediador programa las jornadas de encuentro junto con el sabedor, en este caso un médico tradicional que trabaja con plantas medicinales; planean y acuerdan su intervención desde el uso que se hace de las mismas, su hábitat, el espacio de uso en el que crecen las características de las hojas y el uso en la medicina ancestral. A su vez, el docente mediador estudia las características taxonómicas y aspectos científicos de estos seres. Así tanto el sabedor, como el alumno y el docente mediador retroalimentan y complementan su saber.

Durante las jornadas de trabajo el docente mediador coevalúa los avances en materia cognitiva y experimental por su parte, con el sabedor aprenden el valor de las cosas, el respeto al sentido espiritual de los elementos y la valoración del colectivo humano que hace parte de su comunidad y que fundamenta su ejercicio en su actuar en comunidad. El estudiante, con los conocimientos adquiridos, respeta la flora que observa, busca estrategias de conservación y promoción del respecto al ambiente y los recursos naturales.

En este compartir de conocimientos y actores se fortalece las relaciones y tejido social y cultural de la comunidad, a su vez que los alumnos hablan sobre los problemas o conflictos relacionados con el recurso plantas medicinales, se plantean actividades de intervención en la comunidad a través de ferias y encuentros donde los alumnos comparten lo aprendido con la comunidad.

En todo este trayecto, los estudiantes todo el tiempo están trabajando en el conocer, en el saber hacer y en el saber ser desde elementos que le son cercanos y que le permiten acercarse al conocimiento universal a partir de lo que tiene a su alrededor. Y no sólo eso, sino que practica el liderazgo, la búsqueda de información primaria y secundaria, la creatividad, y la resolución de problemas de su entorno.

Durante el proceso es clave la valoración de la comunidad que coevalúa el desempeño actitudinal de los alumnos fuera del aula, para favorecer la integralidad en la formación de modo que responda a los principios y valores tradicionales.

El sistema de solución contempla también la socialización de informes del desempeño académico y actitudinal obtenidos durante el periodo académico ante los conocedores y sabedores donde es el mismo alumno quien describe cómo se llevó a cabo su proceso académico, las fortalezas, las dificultades en el tránsito y los compromisos para llegar a la meta de aprendizaje. A su vez, el estudiante socializa su autoevaluación en el contexto comunitario; es allí, donde los sabedores en su devolución, rebatirán o reafirmarán lo expresado por el estudiante, pues son testigos y mediadores en el alcance de los principios y valores en la comunidad.

Para lograr en la práctica parte de este cambio, se utiliza un instrumento de Registro de Actividades, que es la bitácora en la que el docente mediador registra los avances del alumno, considerando la opinión que tiene cada sabedor del desempeño actitudinal de los alumnos en formación. El registro de actividades va acompañado de un proceso de planificación bien pensado y enfocado hacia el saber y saber hacer, y hacia las necesidades particulares de cada individuo, donde las actividades estén diseñadas con un enfoque que estimule la creación, la colaboración, la comunicación y la lúdica.

A medida que se va avanzando en el desarrollo de las actividades proyectadas para alcanzar los aprendizajes, tanto el docente-mediador, como el mismo estudiante, van registrando en la planilla la valoración determinada y además se valen de sus diarios de trabajo para registrar reflexiones personales a partir de los diálogos en los encuentros intergeneracionales con los sabedores, también describen sus procesos, tanto desde el 
rol de mediador como el de alumno. Aquí también es importante el diálogo entre el Sabedor y el estudiante quienes comparten experiencias, enseñanzas y pensamientos referentes a lo aprendido.

Es clave indicar que los mecanismos de comunicación entre el docente-mediador, el alumno y la comunidad debe ser efectivos, donde exista la confianza y la objetividad y la empatía para que el alumno exprese de manera oral y/o escrita sus avances, inquietudes y necesidades respecto del proceso.

La valoración de los estudiantes es un proceso que se realiza en doble vía, primero, el conocimiento académico que se evidencia en la adquisición de competencias básicas y específicas de acuerdo con el plan curricular propuesto, y, por otro lado, la valoración de la comunidad, quien valida la conducta en sociedad. Así, el alumno es acompañado y valorado en el trato con las personas, en el cumplimiento de sus responsabilidades en el hogar, en el respeto a sus mayores, en las buenas costumbres, en la participación comunitaria, entre otras.

Para validar el prototipo y poder demostrar que la articulación entre la comunidad y la escuela es generadora de aprendizajes para la vida se realizó acercamientos para obtener información primaria; en este punto fue crucial conocer de la comunidad la respuesta a la siguiente pregunta: ¿Qué tipo de estudiante queremos egresar de la escuela? La información obtenida dio luces para proponer la metodología de la articulación entre la comunidad y la escuela. La manera como se realizó el proceso facilitó obtener muy buenos resultados durante los testeos realizados con los diferentes grupos focales, utilizando diversas técnicas relacionadas con el Design Thinking tales como: más/mejor, mapa de empatía, diagrama de ERAF y los 5 porqués.

El conjunto de las acciones y dinámicas en las que la escuela y la comunidad se articulan para cumplir con el objeto de la educación de manera contextualizada es a lo que se le denomina enfoque etnoeductivo de comunidad-escuela, tal cual lo planeta (Riascos, 2021)

\section{Resultados}

Los pueblos de comunidades negras ubicados en territorios étnicos comprenden que la educación es el mecanismo por excelencia para reproducir, conservar y construir la cultura; permite generar conciencia colectiva, respeto por la vida, establecer formas de relacionamiento armónico con el medio para construir sociedades y evolucionar.

El impacto que genera una educación pertinente al contexto étnico (etnoeducación), que dialogue con la cosmovisión de los pueblos, que reafirme la identidad individual y colectiva y sea sensible a los símbolos, significados, interpretación de la realidad y visión de futuro, impactan de manera muy positiva en el bienestar de estas comunidades, al reducir las brechas educativas y favorecer la inclusión de todos los ciudadanos en igualdad de condiciones y oportunidades para convivir.

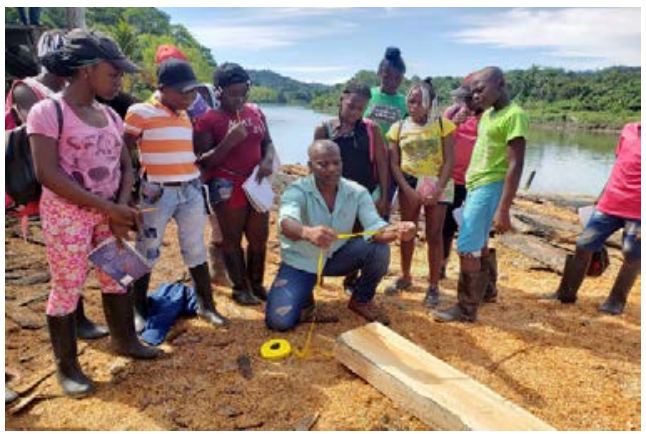

Figura 4.

Clase de etno-matemática desde el enfoque de comunidad-escuela. Aprendizaje en contexto. Cálculo de volumen de madera.

La forma de evaluar el impacto de la iniciativa es precisamente a través de la validación que hace la comunidad como centro de la cultura y sociedad educadora, entendiendo que es quien acompaña y orienta el proceso formativo que la comunidadescuela debe desarrollar en el alumno.

Una de las fortalezas en la evaluación y seguimiento al proyecto que se ejecuta, es el nivel de fortalecimiento organizativo y liderazgo de la comunidad, los planes de vida colectivo dentro del territorio, orientados hacia la defensa y conservación del territorio con todo su acervo cultural; esto ha favorecido que las iniciativas de transformación educativa sean apoyadas por los aliados estratégicos y las dinámicas de orden local, nacional a través de la normatividad e internacional con acciones de gran impacto como la Agenda 2030.

Los resultados obtenidos han sido entre otros los siguientes:

- Se ha podido influir en los pequeños pero significativos retos en materia educativa a nivel local y nacional a través de la participación en convocatorias de experiencias exitosas en el aula donde se ha logrado reconocimientos que motivan a la comunidad educativa a seguir avanzando en la propuesta educativa. (se recomienda ver video de evidencia de experiencia exitosa https://www.youtube.com/watch?v=QkQqRCqg-

WA\& $\mathrm{t}=6 \mathrm{~s}$

- Se empieza a evidenciar mejoramiento en los resultados de las pruebas nacionales en los niveles superiores.

- La organización política comunitaria se ha articulado al proceso educativo, mediante la incorporación de acciones que dan relevancia al Proyecto Etnoeducativo Comunitario (PEC) que se está desarrollando.

- Los docentes han adquirido gran variedad de herramientas pedagógicas y metodológicas relacionadas con la educación inclusiva y contextualizada.

- Se ha logrado incluir metodologías relacionadas con la escuela del siglo XXI y se refleja en los alumnos y egresados habilidades desarrolladas y talentos potenciados a través de las diversas actividades escolares que involucran a la comunidad.

- Se encuentran en diseño y ejecución una gran variedad de iniciativas docentes encaminadas hacia la adquisición de aprendizajes a través de actividades de servicio a la comunidad. 
- La iniciativa es atractiva para aplicarse en otros territorios, pueblos y/o comunidades pequeñas.

\section{CONCLUSIONES}

Sostenibilidad:

El principal mecanismo de sostenibilidad del proyecto está dado por el nivel organizativo de la comunidad y su capacidad de gestión y de proponer iniciativas que vayan en pro del proyecto de vida colectivo y la defensa de la identidad y el territorio ancestral. Un aspecto de gran relevancia es el hecho de estar organizados por Consejos Comunitarios a partir de la ley 70 de 1993, que le atribuye derechos y deberes de administrar el territorio y favorecer la conservación y desarrollo de la comunidad desde sus propias realidades.

Transferibilidad a otros contextos:

Si observamos el Colegio ubicado en la vereda del TRANAL del Municipio de Silvia-Cauca, la institución Educativa Técnica El Tranal a través del universo Misak y del planeamiento educativo Guambiano (PEG), tiene como misión "formar y educar al ser Misak, multilingüe e intercultural; mediante el trabajo material, intelectual, investigativo y creativo, con sentido de pertenencia, generando procesos individuales y comunitarios con capacidad de manejo adecuado de los recursos naturales y el medio ambiente: enfrentándose crítica y autónomamente frente a sí mismo, a los demás, a la naturaleza y a la modernidad forjando su propio destino".

De acuerdo con lo anterior, podemos demostrar que en Colombia existe un gran número de grupos étnicos o culturalmente diferenciados, pertenecientes a comunidades negras e indígenas, que igualmente buscan estrategias para acceder a una educación de calidad, contextualizada y que reduzca las brechas que dificultan el desarrollo de sus pueblos.
Estas características tan similares en estas comunidades permiten que la iniciativa sea fácilmente implementada en sus territorios y puedan obtener los resultados esperados.

Recomendaciones de aplicación.

Las recomendaciones de aplicación están relacionadas primeramente, con la decisión de las comunidades de priorizar la educación en sus territorios, segundo, debe haber un proceso organizativo y de unanimidad en la visión de desarrollo y territorio que se tiene como comunidad: ese empoderamiento permitirá enfilar acciones planeadas que apunten a la visión que se ha propuesto.

Es recomendable que exista apoyo deliberado de las entidades territoriales como alcaldías, gobernaciones e instituciones del Estado que acompañen el proceso en su diseño, desarrollo y evaluación. Desde allí, se deben adaptar los procesos a las particularidades culturales de las comunidades que desean implementar este modelo de educación contextualizada.

\section{REFERENCIAS}

Colombia, M. d. (s.f.). Página web Ministerio del Interior de Colombia. Obtenido

https://www.mininterior.gov.co/lainstitucion/normatividad/ley-70-de-1993-agosto-27-porla-cual-se-desarrolla-el-articulo-transitorio-55-de-laconstitucion-politica

Marles, L. A. (1997). La etnoeducación: una dimensión de trabajo para la educación en comunidades indígenas de Colombia. Revista Iberoamericana de Educación, 12. Obtenido de https://rieoei.org/RIE/article/view/1136

Riascos, B. O. (2021). Proyecto Etnoeducativo Comunitario de la Comunidad-Escuela. Cali-Valle del cauca. 